Revue d'histoire de l'enfance « irrégulière »

Le Temps de l'histoire

5 | 2003

Pratiques éducatives et systèmes judiciaires

\title{
Les colonies pénitentiaires pour jeunes détenus : des établissements irréformables (1850-1914)
}

Éric Pierre

\section{(2) OpenEdition \\ Journals}

Édition électronique

URL : http://journals.openedition.org/rhei/891

DOI : 10.4000/rhei.891

ISBN : 978-2-7535-1643-4

ISSN : $1777-540 \mathrm{X}$

Éditeur

Presses universitaires de Rennes

Édition imprimée

Date de publication : 15 novembre 2003

Pagination : 43-60

ISSN : 1287-2431

Référence électronique

Éric Pierre, «Les colonies pénitentiaires pour jeunes détenus : des établissements

irréformables (1850-1914) », Revue d'histoire de l'enfance « irrégulière » [En ligne], 5 | 2003, mis en ligne le 02 juin 2007, consulté le 03 décembre 2020. URL : http://journals.openedition.org/rhei/891 ; DOI : https://doi.org/10.4000/rhei.891 


\section{Les colonies pénitentiaires pour jeunes détenus : des établissements irréformables (1850-1914)}

Tout chercheur travaillant sur l'histoire des établissements correctionnels français, du XIXème siècle aux années 1970, ne peut qu'être saisi à la fois par la rudesse des conditions d'existence des jeunes détenus qui y séjournent, par le poids de l'immobilisme qui règne dans ces établissements, et plus largement dans l'ensemble du secteur de l'enfance de justice. À tel point que s'il fallait choisir un trait majeur pour caractériser les évolutions de l'éducation correctionnelle en France, organisée surtout autour des colonies agricoles, c'est sans doute les mots d'inertie, de stagnation et de paralysie qui viendraient les premiers à l'esprit. Ayant mené des études monographiques sur plusieurs établissements privés ou publics, ayant suivi l'évolution de certains d'entre eux sur près de cent années - du milieu du XIXème siècle aux années 1930 -, nous avons toujours été marqués par la faiblesse des changements dans leur régime intérieur, dans leur organisation, dans les méthodes éducatives et techniques coercitives qui y sont développées, ainsi, surtout, que dans les conceptions correctives sur lesquelles ils reposent. En travaillant sur la colonie agricole et pénitentiaire de Mettray, dont l'histoire est emblématique de celle des colonies privées, nous avons été surpris du poids des permanences : des règles édictées lors de son ouverture en 1839 restent encore en vigueur lors de sa fermeture, décidée par le ministère de la Justice en 1937, à la suite des campagnes de presse contre les bagnes d'enfants. Les comparaisons internationales nous ont aussi indiqué que s'il existait partout des résistances institutionnelles, elles étaient sans doute plus fortes en France que dans d'autres pays. ${ }^{(2)}$ La Belgique, par exemple, réussit plus vite la transformation de ses établissements correctifs, en soustrayant les jeunes au système pénal, en promouvant le
Éric

Pierre $^{(1)}$

(1) Historien, maître de conférences, HIRÈS, université d'Angers.

(2) Marie-Sylvie

Dupont-Bouchat et Éric Pierre, [dir.], Enfance et justice au XIXème siècle. Essai d'histoire comparée de la protection de l'enfance 1820-1914, Paris, PUF, 2001, 443 p.

Éric Pierre / p. 43 à 60 
(3) Marie-Sylvie

Dupont-Bouchat, De la prison à l'école. Les pénitenciers pour enfants en Belgique au XIXème siècle (1840-1914), Louvain-laNeuve, UGA, 1996.

(4) Sur ce sujet, voir Éric Pierre, "Juge de papier, juge de chair. Du tribunal pour enfants et adolescents au juge des enfants (1880-1951) ", in Monique Charvin, JeanFrançois Gazeau, Éric Pierre, Françoise Tétard, Recherche sur le juge des enfants. Approches historique, démographique, sociologique. Rapport de recherche, ministère de la Justice (France), 1996, p. 10-63. Voir aussi dans ce numéro, les articles de Jean Trépanier et David Niget.

(5) Pour le XXème siècle, voir Antoine Prost, « La famille et l'individu ", in Philippe Ariès et Georges Duby, Histoire de la vie privée, patronage, et en mettant au point les nouvelles techniques d'observation des mineurs délinquants. ${ }^{(3)}$ À tel point, que dès les années 1910 , ce pays devient un modèle pour les réformateurs français, mais un modèle inaccessible. Dans les années 1930, le journaliste Alexis Danan, principal contempteur des établissements pour jeunes, prend encore pour modèle les institutions belges.

Nous ne pouvons donc qu'être surpris de la permanence des établissements français, de leur capacité à ne pas évoluer, à ne pas réagir aux mutations de la société. Alors que la justice des mineurs se modifie, que des tribunaux pour enfants et adolescents sont créés en 1912, prenant en compte la spécificité judiciaire de la jeunesse, ${ }^{(4)}$ que la psychologie de l'enfance se construit comme science, les établissements font preuve d'une inertie presque absolue. Ces derniers semblent relativement indifférents aux transformations qui touchent le secteur dans lequel ils se situent, mais aussi aux évolutions concernant le statut de l'enfant dans la société française. ${ }^{(5)}$ Alors que tout semble évoluer, que la place des jeunes dans leur famille se modifie profondément, les établissements restent immuables.

Il nous faut donc chercher dans l'histoire de l'éducation correctionnelle en France ce qui peut permettre de comprendre cet immobilisme. Les causes de ce phénomène apparaissent variées. Certaines se retrouvent en continuité sur l'ensemble de notre période, telles la méfiance du personnel devant toute innovation à l'intérieur des établissements, qui pourrait remettre en cause des habitudes acquises et des modes de domination exercés sur les jeunes, l'indifférence du pouvoir législatif envers un secteur relativement marginal, et surtout l'absence de moyens budgétaires. ${ }^{(6)}$ Mais la part relative de ces éléments change suivant les périodes et, plus épisodiquement, d'autres phénomènes interviennent de façon parfois décisive. En schématisant, nous pouvons distinguer deux phases qui se chevauchent en partie. La première débute avec le Second Empire et se prolonge sur plus de soixante ans. Pendant cette période, l'Administration pénitentiaire, blessée d'avoir été écartée de la gestion des établissements au profit de l'initiative privée, tente de reprendre la direction du secteur de l'enfance délinquante. Elle veut s'en 
assurer le contrôle, en privilégiant ses colonies au détriment de celles du privé. Peu importe alors que les établissements n'évoluent pas, du moment qu'elle réussit à affirmer son pouvoir et à reprendre la maîtrise du secteur. Pendant de longues années, l'Administration mène une véritable guérilla qui se fait au détriment des jeunes. La seconde période commence après la défaite de 1870-1871 et dure une trentaine d'années. La justice des mineurs est alors un terrain d'étude et d'action privilégié des réformateurs des prisons, des spécialistes de la question pénitentiaire qui se réunissent dans des sociétés de plus en plus nombreuses : "Société générale des prisons", "Comité de défense des enfants traduits en justice", "Union nationale du patronage". Ils élaborent alors un nombre impressionnant de programmes de réforme des établissements. Cependant, ceux-ci échouent en raison de l'absence de volonté politique du gouvernement, de moyens budgétaires, et devant l'indifférence de l'Administration pénitentiaire. Les grands projets de réforme débouchent tout au plus sur des réformettes. L'éducation en maison de correction devient alors un secteur abandonné (d'autant plus que des alternatives à l'enfermement apparaissent), ultime solution pour les enfants les plus difficiles ou des familles les plus instables. Les colonies ne sont plus considérées que comme un mal nécessaire, bien loin de la vocation éducative pourtant mentionnée lors de leur création.

\section{L’Administration pénitentiaire contre les établissements privés} (1850-1914) : une victoire à la Pyrrhus

En France, à la fin des années 1840, la détention en colonie agricole s'impose comme mode d'éducation spécifique de la jeunesse délinquante. À la suite des débats pénitentiaires virulents de la monarchie de Juillet, (7) l'enfermement des jeunes dans des établissements particuliers, situés à la campagne, et dans lesquels ils doivent recevoir une éducation morale, religieuse, et professionnelle est promu. L'emprisonnement cellulaire intégral et l'emprisonnement mixte (cellule de nuit/vie en commun de jour) sont officiellement abandonnés. ${ }^{(8)} \mathrm{Il}$ en est de même des tentatives de confier les jeunes acquittés de l'article 66 du Code pénal à des familles de paysans et d'artisans. Il s'agit alors de sortir les jeunes des volume 5, Paris, Seuil, 1987, p. 61-113.

(6) L'étude des budgets de l'éducation correctionnelle reste à faire. Un rapide survol des débats parlementaires lors de la présentation par le gouvernement des budgets des ministères de l'Intérieur, puis de la Justice, montre le faible intérêt des élus pour la question des jeunes détenus.

(7) Jacques-Guy Petit, Ces peines obscures, la prison pénale en France 17801880, Paris, Fayard, 1990, 749 p.

(8) Ce qui n'empêche pas les anciennes prisons de continuer à fonctionner, comme celle de la Petite Roquette à Paris. Michelle Perrot, «Les enfants de la Petite-Roquette ", L'Histoire, $\mathrm{n}^{\circ} 100$, mai 1987, p. 30-38. 
(9) Politique exprimée dans la circulaire du ministre de l'Intérieur Duchâtel en 1840 et dans le transfert des frais d'entretien des jeunes détenus du budget des départements à celui de l’État, en 1841. Éric Pierre, « Débats pénitentiaires, politiques correctionnelles et vote de la loi de 1850 ", in Michel Chauvière, Pierre Lenoël et Éric Pierre (s.d.),

Protéger l'enfant. Raison juridique et pratiques sociojudiciaires (XIXème-

XXème siècles), Rennes, Presses universitaire de Rennes, 1996, p. 71-105.

(10) Sur la création de ces établissements : Henri Gaillac, Les maisons de correction 18301945, Paris, Cujas, 1991 (2ème édition), 463 p. Christian Carlier, $\mathrm{La}$ prison aux champs. Les colonies d'enfants délinquants du nord de la France au XIXème siècle, Paris, L'Atelier, 1994, 734 p. prisons, de leur donner une formation professionnelle leur permettant de trouver un travail dans le monde rural, et de les moraliser dans ces nouveaux établissements. Il s'agit aussi de gérer une politique répressive qui, à partir du début des années 1840, aboutit à une forte croissance des envois en correction..$^{(9)}$ De ce point de vue, la colonie agricole semble bien mieux adaptée à l'accueil des gros contingents de jeunes détenus que la prison. ${ }^{(10)}$

La loi de 1850 votée par l'assemblée législative à majorité conservatrice de la seconde République, dans un climat de peur sociale fortement ressenti depuis les événements de juin 1848, ${ }^{(11)}$ vient confirmer a posteriori, l'adoption de la colonie pénitentiaire agricole comme établissement de correction. ${ }^{(12)}$ Soucieux de ne pas mettre en place un système d'éducation "nationale" qui vient d'être refusé aux enfants du peuple, et sous des arguments économiques (le privé coûte moins cher que le public) et éducatifs (les fonctionnaires ne peuvent être des éducateurs dévoués), les représentants offrent même un droit de priorité aux établissements privés sur ceux bâtis par le gouvernement. La loi incite fortement les entrepreneurs à ouvrir des colonies en échange d'un prix de trousseau et d'un prix de journée versés par le ministère de l'Intérieur pour chaque enfant accueilli. Elle précise encore que c'est seulement dans le cas où les réalisations de l'initiative privée s'avèreront insuffisantes que l'État pourra ouvrir ses propres établissements. Ce dernier ne doit donc intervenir que de façon subsidiaire à l'initiative privée.

Logiquement, le vote de la loi donne naissance à une seconde vague d'ouverture de colonies agricoles privées, ainsi qu'au développement des anciennes colonies qui avaient été bâties avant la loi. Des particuliers, des associations ou des congrégations profitent du délai de cinq ans prévu par la loi pour négocier avec l'Administration la fondation de nouveaux établissements. Entre 1851 et 1856, plus d'une dizaine de colonies ouvrent. Pour les filles, l'ordre du Bon-Pasteur d'Angers met en place à l'échelle nationale un véritable réseau d'institutions. Un parc d'établissements privés est ainsi progressivement créé.

La loi de 1850 marque donc pour plus de cent ans l'éducation correctionnelle des jeunes. Et cela dans trois domaines au moins : d'abord 
en favorisant l'enfermement massif des jeunes, alors que des alternatives telles que le patronage ou l'accueil par des familles avaient été envisagées, ensuite par le choix essentiellement idéologique du travail agricole alors que l'essentiel de la clientèle des maisons de correction provient du monde urbain, enfin par la primauté accordée à l'initiative privée sur les institutions du gouvernement.

\section{Une sourde hostilité}

Or l'Administration pénitentiaire accepte mal cet état de fait. ${ }^{(13)}$ Pour certains de ses membres influents, tels Charles Lucas, l'État délègue au privé une partie de son droit de punition, ce qui est largement inacceptable. De plus, l'Administration estime que la loi de 1850 est le fruit d'une situation politique exceptionnelle, celle d'une période de transition, et qu'elle ne reflète pas les débats pénitentiaires des années précédentes plutôt favorables à la fin de la monarchie de Juillet à l'enfermement cellulaire. ${ }^{(14)}$ Mais le régime autoritaire en place à partir du 2 décembre 1851 ne favorise pas l'expression des dissensions administratives. L'heure est à la discrétion. Ainsi, les premières années des colonies agricoles privées sont bien marquées par l'opposition de l'Administration pénitentiaire, mais sous la forme d'une sourde hostilité. Pendant cette période, l'Administration utilise abondamment les colonies privées, en raison de la forte augmentation des populations détenues, tout en entretenant une attitude de méfiance à leur égard. L'Administration des prisons s'est vue imposer la primauté accordée aux colonies privées, et elle se doit de l'accepter. Cependant, dès 1854, F. Persigny, le ministre de l'Intérieur, signant un texte écrit par le directeur de l'Administration pénitentiaire, L. Perrot, déplore cette primauté. En fait, il reprend les craintes exprimées l'année précédente par l'inspecteur général adjoint Bucquet.

En effet, dès 1853, ce dernier dresse un tableau contrasté des établissements correctionnels, ${ }^{(15)}$ développant déjà plusieurs thèmes qui seront abondamment utilisés pour mener campagne contre le privé : l'absence de règles strictes de comptabilité et d'administration dans la plupart des colonies, la mauvaise tenue des registres, le coût plus élevé du prix de journée attribué aux établissements privés par rapport aux dépenses des

(11) Maurice Agulhon, 1848 ou l'apprentissage de la République, Paris, Seuil, 1973, 254 p.

(12) Du moins pour les garçons.

(13) Jean-Marie

Renouard a largement mis à jour ce conflit dans De l'enfant coupable à l'enfant inadapté. Le traitement social et politique de la déviance, Paris, Paidos Centurion, 1990, 199 p.

(14) Sur ce point, voir l'introduction de Michelle Perrot au tome 4 des Euvres complètes d'Alexis de Tocqueville, Paris, Gallimard, 1984.

(15) Paul Bucquet, Tableau de la situation morale et matérielle en France des jeunes détenus et des jeunes libérés, et Recherche statistique sur les colonies agricoles, les établissements correctionnels et les sociétés de patronage 
de jeunes détenus, Paris, Imprimerie administrative, 1853. établissements publics, et surtout l'insuffisance et l'incapacité du personnel. Bucquet juge cependant satisfaisants le régime alimentaire et le bien-être matériel accordé aux colons, ainsi que les conditions d'hygiène et l'état sanitaire général des colonies. Le premier tableau fait par l'inspecteur des prisons présente plusieurs ambiguïtés. Il reconnaît des mérites aux établissements privés mais, en même temps, il insiste sur le poids que leurs dépenses d'entretien font peser sur le budget de l'État. En fait, nous sentons qu'il n'ose pas encore exprimer totalement toutes ses réserves sur le compte des établissements privés.

Les années suivantes voient les critiques contre les établissements privés se manifester plus ouvertement, surtout dans les rapports non publiés des inspecteurs des prisons. L'absence des archives de l'Administration pénitentiaire ne permet pas de suivre précisément ce phénomène, mais il semble bien que les inspecteurs soient beaucoup plus critiques avec les colonies que ne le sont les autres fonctionnaires. De leurs inspections, ils rapportent des descriptions souvent terribles sur les conditions de vie des colons, sur la violence des moyens disciplinaires, sur l'état d'insalubrité des colonies, sur leur inorganisation administrative, sur les désordres multiples qui y règnent, etc. Le plus fréquemment, ces rapports sommeillent jusqu'à la prochaine inspection. Le ministre et le directeur de l'Administration pénitentiaire hésitent à fermer des établissements, même si les conditions d'existence des jeunes y sont déplorables. Car l'Administration, hostile par principe à l'envoi de jeunes détenus dans des établissements privés, continue à trouver dans leur existence un outil de gestion souple des populations pénales. Elle ne manque pas ainsi de places. Elle peut, en fermant ou en facilitant l'ouverture de nouvelles colonies, anticiper les flux de populations. Elle peut aussi en choisissant un projet plutôt qu'un autre favoriser certaines orientations.

Cela explique, en partie, les contradictions apparentes de l'Administration, qui oscille alors entre une attitude fréquemment défavorable, et un recours permanent aux "services du privé". Il faudrait aussi apporter une nuance en fonction des ministres et des directeurs de l'Administration. Les passages de Persigny à l'Intérieur semblent être les périodes les plus défavorables au privé. Mais de toute façon, quel que soit leur 
degré d'hostilité, tous les inspecteurs, tous les fonctionnaires souhaitent accroitre le contrôle sur les établissements privés, et limiter leur part d'autonomie. ${ }^{(1)}$ Pour cela, l'Administration utilise des moyens de pression discrets : gestion du flux des entrants dans tel ou tel établissement, fixation des prix de journée, paiement irrégulier des sommes dues aux colonies, inspections plus ou moins critiques, etc. Ensuite, le renforcement du nombre d'établissements publics, permanent sous le Second Empire et sous la IIIème République, permet à l'Administration de ne pas dépendre totalement du secteur privé. ${ }^{(17)}$ Mais c'est l'élaboration d'un règlement national applicable dans toutes les colonies privées qui forme la clef de voûte de la reprise en main du secteur par l'Administration. Ce projet existe depuis $1853{ }^{(18)}$ Il provoque alors l'opposition des directeurs d'œuvres, attachés à leur indépendance et suffisamment puissants pour obtenir son rejet. Après un premier règlement national "provisoire" élaboré en 1864, qui soulève encore l'hostilité des directeurs, et un nouveau recul de l'Administration, un second règlement intervient en 1869. Très pointilleux, il est définitivement adopté. L'inspection dispose désormais de normes fixes et précises, surtout en ce qui concerne les conditions matérielles à faire respecter par les directeurs : par exemple, le nombre de $\mathrm{m} 3$ dont chaque enfant doit disposer dans les dortoirs, le personnel nécessaire en fonction de l'effectif des jeunes détenus, les vêtements obligatoires, l'organisation des différents services, etc. Enfin, toutes les formes de châtiments corporels deviennent interdites. L'Administration voit donc à la fin du Second Empire son pouvoir de contrôle notablement accru. Une partie de ses ambitions est ainsi satisfaite. Elle dispose d'un nouveau moyen de pression, et n'hésite pas alors à l'utiliser pour faire fermer des colonies.

Dans cette démarche, le bien-être des jeunes détenus n'est en aucun cas sa préoccupation prioritaire. Il apparait évident que ce règlement n'a pas été élaboré pour améliorer leurs conditions de vie, même si plusieurs articles peuvent leur être favorables, mais simplement pour disposer d'un outil de contrôle sur les colonies. Il n'est d'ailleurs pas question d'appliquer ce règlement dans les établissements publics. Ce dernier est une arme dans les mains des inspecteurs et de l'Administration,

(16) Dans un autre texte, nous faisons le postulat que l'Administration dispose cependant de suffisamment de moyens de contrôle sur les œuvres privées pour leur imposer ses vues principales. La réaction d'hostilité de

l'Administration relève alors, suivant les personnes, soit d'un sens élevé du rôle de l'État et de ses fonctions régaliennes, soit d'une susceptibilité blessée. Éric Pierre, « F.-A. Demetz et la colonie agricole de Mettray entre réformisme "romantique" et injonctions administratives ", Padagogica Historica, numéro spécial « Doers », XXXVIII, $\mathrm{n}^{\circ}$ 2-3, 2002, p. 451-466.

(17) Henri Gaillac, op . cit.

(18) Archives départementales d'Indre-etLoire. Archives de la colonie de Mettray. 
Correspondance de F.-A.

Demetz, volume 7 .

Lettre à Delessert, 5

décembre 1853.

(19) Archives départementales d'Indre-etLoire. Archives de la colonie de Mettray. Correspondance de F.-

A. Demetz.

(20) Éric Baratay,

"Affaire de mœurs, conflits de pouvoir et anticléricalisme : la fin de la congrégation des Frères de Saint-Joseph en 1888 ", Revue d'histoire de l'Église de France, tome 84 ( $\left.\mathrm{n}^{\circ} 213\right)$, juillet-décembre 1998, p. 299-322.

(21) L. Herbette, « L'auvre pénitentiaire. Etude présentée à l'occasion de l'organisation du Musée spécial de l'Administration pénitentiaire ", Melun, Imprimerie administrative, 1891, cité par JeanMarie Renouard, op. cit., p. $55-56$.
(22) Cette dernière colonie a une vocation industrielle. Henri Gaillac, op. cit., p. 161 et p. 163.

(23) D’autres établissements connaissent une existence plus éphémère : Villeneuvesur-Lot qui ne fonctionne que durant deux années ; la colonie de Saint-Bernard, fermée à la suite d'incidents en 1888 , puis réouverte sous la forme d'une colonie industrielle entre 1910 et 1914 . Il existe une quinzaine d'établissements publics, toutes catégories confondues, en 1913, soit 5 de plus qu'en 1871 et 4 de plus qu'en 1881. Cependant, il s'agit surtout de colonies aux effectifs importants, alors que de nombreux quartiers correctionnels, situés dans des prisons et donc aux effectifs plus réduits, ont été fermés. Henri Gaillac, op. cit., p. 261. qui peuvent désormais faire peser une menace permanente sur les directeurs d'établissements. Ceuxci ont bien conscience de la fragilité de leur situation. La correspondance de F.-A. Demetz, le fondateur de Mettray, pourtant la plus célèbre et la plus puissante des colonies privées, montre à de nombreuses reprises sa crainte récurrente de la fermeture de son œuvre. ${ }^{(19)}$

\section{Une défiance affichée}

Si la période politiquement complexe des débuts de la IIIème République voit, par moment, le retour en force des partisans des colonies privées, en particulier à la chambre des députés, l'installation définitive des républicains à partir de 1877 libère l'hostilité de l'Administration à leur égard. Dans les années 1880, l'affrontement entre le pouvoir républicain et l'Église catholique permet que soient révélés dans les presses nationales et locales certains scandales de mauvais traitements à enfants mettant en cause différentes institutions proches des milieux catholiques, quand elles ne sont pas sous la direction directe de congrégations. Ainsi, Cîteaux ferme en 1888 à la suite d'accusations (brutalité et lubricité aggravée) portées contre les Frères par un jeune évadé, et d'une campagne de presse contre "Cîteaux Sodome $"{ }^{(20)}$ Le député républicain de la circonscription de Mettray dénonce l'usage de châtiments corporels. Les Sours du Bon-Pasteur sont également mises en cause dans différentes affaires.

Le pouvoir politique profite d'incidents et de dénonciations de colons pour obtenir la fermeture de colonies. En 1889, Herbette, le directeur de l'Administration pénitentiaire, affirme avec force la primau- 
té du secteur public dès lors qu'il s'agit de punir : «Que tous les services ayant pour objet la privation de la liberté, toutes œuvres de punition ou de correction à exercer sur les personnes, doivent demeurer sous les mains des représentants de l'État. » ${ }^{(21)}$ En conformité avec cette politique, l'Administration poursuit ses créations d'établissements avec le rachat du Val-d'Yèvre et la fondation de Saint-Maurice en 1872, puis surtout l'ouverture de Belle-Ile-en-Mer en 1880, ainsi que d'Aniane en 1886. ${ }^{(22)}$ Suite à ces dernières créations, et à des fermetures de colonies privées, le rapport de force entre les établissements publics et privés s'inverse. En 1889, pour la première fois, plus de garçons en correction sont détenus dans les premiers (2.616 soit 55,83 \%) que dans les seconds (2.072, soit 44,17 \%). Pendant quelques années, les établissements privés redeviendront très légèrement dominants, mais à partir de la fin du siècle, les institutions publiques assurent définitivement et, de plus en plus largement, leur domination. Il faut dire que ces années sont encore marquées par des ouvertures d'établissements publics, et, surtout, les fermetures accélérées d'établissements privés. Parmi les premières, mentionnons l'importante colonie agricole et industrielle d'Auberive en 1897, Cadillac et Clermont-de-l'Oise pour les filles, Birkadem en Algérie en 1908, ainsi que les deux colonies correctionnelles d'Eysses (1895) et de Gaillon (1908). ${ }^{(23)}$ Comme l'Administration pénitentiaire réserve en priorité les enfants des articles 66 et 67 du Code pénal à ses propres établissements, les effectifs des établissements privés s'effondrent, entraînant leur disparition progressive. ${ }^{(24)}$

Cette évolution n'est pas sans susciter des réactions hostiles dans le monde des œuvres. En 1907, devant le Comité de défense des enfants traduits en justice, Robert Georges Picot dénonce « la défiance manifestée par les pouvoirs publics à l'égard des colonies pénitentiaires privées ". ${ }^{(25)}$ Selon lui, la baisse du nombre de jeunes menace, à court terme, d'entraîner la fermeture des dernières colonies privées. Robert Georges Picot accuse les pouvoirs publics d'être animés par un esprit de querelle religieuse. Il prend la défense des colonies, qui répondent à certains besoins, et qui, selon lui, ont su s'adapter aux nouvelles exigences et aux "mœurs nouvelles" : elles possèdent des ateliers industriels, pratiquent
(24) $92 \%$ de diminution des effectifs entre 1875 et 1912. Jean-Marie Renouard, op. cit., p. 56.

(25) Robert-Georges Picot, De la défiance manifestée par les pouvoirs publics à l'égard des colonies pénitentiaires privées, rapport présenté à la séance de 10 avril $1907 d u$ Comité de défense des enfants traduits en justice de Paris, Paris, Kugelman, 1907, 28 p. 
(26) Il en existait 56 en 1880 . la séparation des colons les plus jeunes, ainsi que le patronage des libérés. Il propose qu'on en revienne à la loi de 1850, faisant des établissements publics les supplétifs des colonies privées. Ce plaidoyer, à contresens des évolutions, reste évidemment sans effet. Les colonies privées sont en déroute : il n'en reste plus que 22 en 1889, 20 en 1895, 18 en 1902, 15 en 1908, 8 en 1912. ${ }^{(26)}$ Après la première guerre mondiale, la présence des colonies privées n'est plus qu'une survivance du passé. Mettray ne peut continuer qu'en recherchant de nouvelles clientèles de jeunes, en particulier auprès de l'Assistance publique. L'initiative privée se réoriente en grande partie vers d'autres types de prise en charge des jeunes de justice, du type patronage. Après plus de soixante ans de combat, le terrain est désormais libre pour la pénitentiaire. Cependant, l'Administration ne profite pas pleinement de son succès, car elle subit alors le mouvement de désaffection général à l'égard des établissements correctionnels, qu'elle a en partie organisé. Si elle semble enfin l'emporter, elle règne désormais sur un monde en déclin. Sa victoire sur le secteur privé est un triomphe à la Pyrrhus, car il existe en effet un mouvement de contagion des critiques, qui englobent désormais aussi bien les établissements publics que ceux relevant du privé. C’est tout le secteur de l'éducation correctionnelle qui se trouve alors dévalorisé.

De ce long combat, l'intérêt des enfants est presque systématiquement absent. Si l'Administration, parfois, dénonce la cupidité des directeurs d'œuvres, ainsi que les privations et les violences subies par les jeunes détenus, ce n'est presque jamais pour les faire cesser. De plus, rien ne prouve que les jeunes soient mieux traités dans les établissements dépendant des ministères. L'intérêt de l'Administration passe toujours avant celui de ses "protégés", qui en principe sont détenus pour recevoir une éducation et non pour subir une peine. Celle-ci dépense toujours plus d'énergie à défendre ses positions qu'à obtenir les moyens financiers et humains nécessaires à une réforme du secteur, pourtant largement souhaitée par les réformateurs et les spécialistes des prisons. 


\section{Grands projets et réformettes}

À partir des années 1870 se développe en effet une critique récurrente des institutions correctionnelles, ainsi qu'une volonté conjointe de les réformer. L'imposante commission parlementaire sur le régime des établissements pénitentiaires, qui fonctionne pendant plusieurs années à partir de 1872, met en lumière les dysfonctionnements des colonies privées. Dans cette commission, les établissements de jeunes détenus font l'objet d'une enquête approfondie. Bien qu'il faille interpréter les résultats de celle-ci avec prudence, en raison de la multiplicité des intervenants (parlementaires, fonctionnaires, spécialistes et praticiens de la prison, magistrats, directeurs d'établissements, etc.) et de la variété des travaux (commission plénière, commissions spécialisées, enquête écrite auprès des tribunaux), qui donnent naissance à une profusion de paroles souvent contradictoires, les jugements négatifs l'emportent largement, surtout pour les établissements privés.

\section{Des projets sans suite}

Dès 1872, le directeur de l'Administration pénitentiaire, Jaillant, déclare : «La plupart des colonies privées fonctionnent avec peine ; les enfants n'ont pas tout ce qui est nécessaire. Il existe bien un règlement qui détermine les obligations du directeur de l'établissement, mais ce règlement n'est pas suivi. » ${ }^{(27)}$ En conclusion de son intervention, il réclame une meilleure application et même un renforcement du règlement de 1869. Ces remarques apparaissent cependant bien modérées en comparaison de celles formulées dans un rapport légèrement postérieur. En effet, le 4 avril 1873, le ministre de l'Intérieur demande à la commission de délibérer sur la question des colonies privées et des colonies publiques. Dans son travail, le rapporteur, Bournat, se livre à une critique acérée des colonies privées et de la loi de 1850. En se revendiquant de l'autorité de Ch. Lucas, il déclare que "la colonie privée a fait son temps et n'a plus aujourd'hui sa raison d'être ". ${ }^{(28)}$ Il reprend à son compte l'idée de l'ancien inspecteur des prisons pour qui seul l'État peut assumer le droit de punir. Si Bournat trouve excessive l'idée de fermer les colonies privées, car il estime qu'il en existe quelques-unes de conve-
(27) Commission d'enquête parlementaire, séance du 21 mai 1872, volume 1, p. 47.

(28) V. Bournat, Rapport présenté à la commission sur les questions suivantes : Doit-on, en principe, préférer pour l'éducation correctionnelle le système des colonies privées à celui des colonies publiques ? Doit-on, en conséquence, maintenir sur ce point les dispositions de la loi du 5 août 1850 ?, Paris, Imprimerie nationale, $1875,31 \mathrm{p}$. 
(29) Dont les réussites ne doivent pas cacher l'échec des colonies privées. Ibid., p. 30-31.

(30) Ibid., p. 23.

(31) Ibid., p. 29-30.

(32) Enquête parlementaire sur le régime des établissements pénitentiaires, Rapport sur e projet de loi relatif à l'éducation et au patronage des jeunes détenus, fait par M. Félix Voisin, Paris, Imprimerie nationale, 1875, tome VIII, 187 p.

(33) Ibid., p. 42. nables ${ }^{(29)}$ (Mettray, Cîteaux, Fontgombault), il pense que de nombreux problèmes viennent de la multiplication des malversations survenues lors des dernières années. Selon lui, il suffit de connaître l'histoire des colonies privées fermées pour comprendre le peu de garanties qu'elles offrent. "Cette histoire des colonies privées supprimées depuis 1850 ou susceptibles de suppression, écrit-il, est pleine de détails lamentables. " ${ }^{\left({ }^{0}\right)}$ Détails que le lecteur ne peut cependant pas connaitre, car la commission, "douloureusement émue ", décide la suppression de ce passage du rapport pour ne pas mettre en cause des personnes. Bournat conclut en demandant l'installation de nouvelles colonies publiques et donc l'inversion du rapport de force prévu par la loi de 1850 : il faut désormais donner la priorité aux établissements publics sur les colonies privées, car "l'éducation correctionnelle est un service public que le législateur et l'Administration ne peuvent abandonner aux élans inégaux, intermittents de la charité ». ${ }^{(31)}$

Sans adopter une position aussi radicale, l'ensemble de la commission décide le principe d'une réforme de la loi de 1850. Félix Voisin a la charge de déposer le Rapport sur le projet de loi relatif à l'éducation et au patronage des jeunes détenus. ${ }^{(32)}$ Dans un long travail, il dresse un bilan critique de l'application de la loi de 1850. Il s'attache spécialement à l'étude de deux points : la primauté de l'initiative privée sur le secteur public et l'exclusivité du travail agricole. Dans ce dernier domaine, le législateur s'est trompé en pensant mettre tous les enfants aux travaux des champs, sans tenir compte de la diversité des situations. S'il faut bien appliquer les jeunes de la campagne aux travaux des champs, il ne peut en être de même pour ceux des villes, et même pour ceux des milieux côtiers : il faut donner à chaque enfant un métier en rapport avec son origine, son "avenir présumable", ses besoins futurs. F. Voisin, poursuivant son œuvre de propagandiste favorable au public, développe un dernier argument : l'existence des colonies d'État entretient une concurrence entre secteurs et permet ainsi de résister aux demandes de subventions des directeurs du privé ; il ne faut donc pas laisser le privé en situation de monopole au « risque de voir le Trésor public en souffrir ". ${ }^{(33)}$ Après cette longue démonstration, F. Voisin peut conclure que l'État ne peut être relégué au second rang dans le domaine de l'éducation des jeunes détenus. 
Il propose donc de réformer la loi sur deux points essentiels : la primauté du privé sur le public et l'application exclusive des jeunes garçons aux travaux agricoles. Il élabore cependant un projet de loi qui dépasse largement ces objectifs et qui touche tous les secteurs de l'éducation correctionnelle. En se limitant aux établissements, il suggère, en partie inspiré par les exemples belge et néerlandais, et contrairement à une certaine tradition française, de distinguer le traitement des enfants acquittés et celui des enfants condamnés. Il faut corriger les seconds dans des "maisons correctionnelles", ${ }^{(34)}$ et élever les premiers dans "des maisons de réforme". Dans ces dernières, les jeunes détenus doivent recevoir une instruction primaire, et une éducation morale, religieuse et professionnelle. Un nombre d'instituteurs suffisant doit faire la classe à un public de jeunes en état d'écouter et d'apprendre, donc préservé de la fatigue d'une longue journée de labeur agricole. Pour les moins âgés, il faut même rendre le " travail accessoire ". La formation professionnelle devra être adaptée à l'origine géographique de l'enfant. Sans rentrer dans les détails du régime intérieur, F. Voisin précise le contenu de ces nouveaux établissements, où les jeunes pourront être élevés en commun ou soumis à la séparation individuelle. Enfin, les maisons de réforme, de préférence de petits établissements de moins de 200 jeunes, peuvent appartenir à l'État ou à des sociétés privées. Mais dans ce cas, l'Administration doit établir un cahier des charges précis, agréer un directeur qui engage sa responsabilité, et exercer un véritable droit de regard : une commission de surveillance et une commission de contrôle devront d'ailleurs suivre précisément l'action du directeur. À l'inverse les "maisons correctionnelles" relèvent uniquement de l'État, car " seuls les établissements publics ont le caractère répressif qui convient à l'exécution de condamnations judiciaires ". ${ }^{(35)}$

Voisin propose donc de réviser la loi de 1850 en organisant une véritable séparation des différentes catégories de détenus, en renforçant les établissements d'État, et en développant le travail industriel. S'il ne rompt pas avec les conceptions traditionnelles de l'éducation correctionnelle, il souhaite que les colonies agricoles privées ne soient plus en position hégémonique dans le système pénitentiaire.
(34) Ils seront détenus dans ces maisons correctionnelles après avoir fait leur temps de prison.

(35) Ibid., p. 151. 
(36) Voir dans le chapitre 4 de notre ouvrage, "Les établissements, critiques et réformes ". Marie-Sylvie DupontBouchat et Éric Pierre, [dir.], Enfance et justice..., op. cit., p. 292-314.

((37) En 1880, les moins de douze ans représentent $36 \%$ des jeunes en correction. Henri Gaillac, op. cit., p. 166.

(38) Journal des débats, 21 août 1892, cité par Henri Gaillac, ibid., p. 169.

(39) Voir le tome 3 de Jacqueline CostaLascoux, La délinquance des jeunes en France, 18251968. Textes législatifs et réglementaires, Paris, Cujas, 1978, 230 p.

(40) François Coppée, Le coupable, Paris, Lemerre, 1897, 327 p.
Ce rapport, pourtant essentiel, n'a pas de suite directe. La loi n'est pas modifiée, les nouveaux établissements proposés par Voisin ne sont pas fondés, l'éducation correctionnelle n'est pas réorganisée. Faute de réforme, de moyens financiers, et d'une volonté politique, l'éducation correctionnelle s'enfonce alors dans la crise. Les colonies agricoles survivent, mais de plus en plus difficilement.

\section{Un secteur à l'abandon}

À la suite de ce travail pionnier, pendant près de trente ans, les plans de réforme des établissements correctionnels se multiplient, toujours sans effets. ${ }^{(36)}$ Le Bulletin de la Société générale des prisons en expose plusieurs. Mais la volonté politique et l'argent manquent toujours. Les idées de transformation plus ou moins radicale de ces maisons de correction se développent dans toutes les instances de réflexions étatiques ou para-étatiques, mais les budgets de l'Administration pénitentiaire ne permettent que des réalisations partielles et inabouties. Un seul exemple suffit à le montrer. Depuis Voisin s'est imposée l'idée d'une classification des jeunes par âge, et de nombreux réformateurs proposent que les établissements se dotent d'une section spéciale dite de "réforme" pour les plus jeunes (jusqu'à douze ans). ${ }^{(37)}$ L'Administration pénitentiaire ouvre en 1891 un "internat approprié" à Chanteloup en Maine-et-Loire. En principe, cette institution doit privilégier l'éducation et l'instruction, devenues obligatoires pour tous au début des années 1880. Des instituteurs suffisamment nombreux se trouvent parmi le personnel. La discipline y est réputée moins sévère qu'ailleurs. Il reste cependant un problème non résolu : que faire des enfants qui atteignent l'âge limite de douze ans ? Aucune autre solution n'est envisagée que leur passage dans les sections supérieures des colonies agricoles. Ainsi, les jeunes de Chanteloup seront placés à Saint-Hilaire et se retrouveront donc dans les établissements que les réformateurs avaient souhaité leur éviter. Dès 1892, Joly exprime ses inquiétudes, dans le Journal des débats du 21 août : " N'estil pas à craindre que l'eau filtrée (à Chanteloup) ne se trouve rejetée dans le tout-à-l'égout ? " ${ }^{(38)}$ Globalement, la classification par âge est donc un nouvel échec. Les résultats ne sont pas meilleurs en ce qui concerne la formation professionnelle, qui malgré une plus grande diversité, reste plus orien- 
tée vers l'exploitation du travail des enfants que vers l'apprentissage d'un métier, la discipline, qui repose encore trop sur les châtiments corporels, les privations de nourriture, et l'isolement en cachot, ainsi que l'éducation et l'instruction, largement négligées. Il existe de bons indicateurs de la détérioration des conditions de vie dans ces établissements : la multiplication des évasions, refus d'obéissance, rébellions individuelles, et plus rarement collectives. Aux yeux des contemporains, les établissements correctionnels fabriquent des récidivistes et de futurs criminels. Surtout, ils commencent à apparaître irréformables.

Faute de pouvoir réaliser un plan d'ensemble, l'Administration se lance dans une production normative tatillonne, qu'elle se garde toujours bien d'appliquer à ses propres établissements. ${ }^{(39)}$ Les circulaires et directives deviennent de plus en plus nombreuses, mais une analyse précise de l'action des préfets et du ministre de l'Intérieur montre que les efforts se relâchent rapidement.

En conséquence, reposant sur leur incapacité à se transformer, la critique des colonies agricoles se diffuse encore largement. Elle touche désormais aussi bien les établissements privés que les établissements publics. L'opprobre qui pèse sur elles sort du cercle étroit des spécialistes. Dans un roman, Le coupable, F. Coppée émeut avec son jeune héros placé dans une colonie corruptrice. ${ }^{(40)}$ Les mots employés par Joly, cités plus haut, montrent bien les représentations négatives des contemporains sur les colonies agricoles, d'autant qu'ils sont ici utilisés par un des plus répressifs des spécialistes des prisons et en principe un des plus favorables aux colonies pénitentiaires. Face à ce flux de critiques, les juges hésitent de plus en plus à envoyer les jeunes délinquants dans ces établissements. ${ }^{(41)}$ Il existe alors un véritable mouvement de désaffection à leur égard. Echaudés pas les échecs successifs de réforme, les spécialistes les plus reconnus des questions pénitentiaires délaissent l'idée de rénovation des colonies, abandonnant l'élaboration des programmes de réforme à des personnalités secondaires du mouvement. ${ }^{(42)}$ Pratiquement, des alternatives apparaissent : avec la loi de 1898, il devient possible de confier des jeunes délinquants à des sociétés de patronage ou à des établissements charitables dépendant de l'Assistance publique. ${ }^{(43)}$ Au-delà du milieu des
(41) Phénomène qui

se vérifie aussi bien dans les statistiques nationales du Compte général de l'administration de la justice criminelle que dans la pratique locale des tribunaux. Éric Pierre, Samuel Boussion, Delphine Gruau, David Niget, Analyse de la délinquance et de la violence des jeunes en Maine-etLoire : Approches historique et archivistique (1870-années 1950), rapport de recherche remis à la direction de la Protection judiciaire de la jeunesse du ministère de la Justice, octobre 2002, 330 p.

(42) Par exemple, Louis Puibaraud, Les maisons d'éducation pénitentiaire et correctionnelle. Essai d'un plan de réforme de la loi du 5 août 1850 sur les jeunes détenus, Paris, Gazette du Palais, 1894, 63 p.

(43) Éric Pierre, « La 
loi de 19 avril 1898 et les institutions ", le Temps de l'bistoire, $\mathrm{n}^{\circ} 2,1999$, p. 113-127.

(44) « Circulaire du Bureau central ", Revue pénitentiaire, 1903, p. 1401-1403.

(45) Michelle Perrot, "Quand la société prend peur de sa jeunesse en France, au 19ème siècle ", Les jeunes et les autres. Contributions des sciences de l'homme à la question des jeunes, Vaucresson, CRIV, 1986, p. 19-27. Il faut aussi mentionner les effets médiatiques du phénomène "Apaches" à Paris. Michelle Perrot, « Dans le Paris de la Belle Époque : les “Apaches”, premières bandes de jeunes ", in Bernard Vincent, Les marginaux et les exclus dans l'histoire. Cabiers Jussieu, $\mathrm{n}^{\circ} 5$, 1979, Paris, UGE, p. 387-406. Texte republié dans Michelle spécialistes, la critique des établissements touche la presse et devient un mouvement d'opinion. Les colonies agricoles semblent se diriger vers leur mort par extinction progressive de leur clientèle, d'autant que les difficultés économiques de la fin du siècle n'améliorent pas les choses.

Les établissements ne trouvent plus l'équilibre budgétaire indispensable à leur survie. Les fermetures de colonies privées, nous l'avons vu, se succèdent à un rythme alors élevé. L'État peu à peu se substitue donc à l'initiative privée défaillante et découragée ; globalement, cette évolution satisfait l'Administration pénitentiaire, mais elle la fragilise aussi. En fait, incapables de se réformer, les colonies vont sans doute mourir.

Cependant, ce mouvement, encouragé depuis les années 1880 par les réformateurs des prisons finit par dépasser leurs espérances, et même par les inquiéter. En 1903, le Bureau central de l'Union des sociétés de patronage, pourtant fondé pour défendre une forme d'alternative à l'enfermement, décide d'intervenir pour changer le cours des choses. Une pétition adressée aux membres de la magistrature, et signée « de tous les présidents d'œuvres et de tous les criminalistes les plus éminents », présente les avantages de l'envoi en correction. Les signataires regrettent que les juges pratiquent trop la remise des enfants à leur famille, les courtes peines de prison, et les placements inopportuns en patronage. "Et puis la maison de correction, écrivent-ils, n'est pas comme on se l'imagine trop facilement, une sorte de bagne pour enfants. Les maisons de correction, les colonies pénitentiaires modernes ressemblent à des écoles; c'est bien à tort qu'on leur fait une mauvaise réputation. ${ }^{(44)}$ Ces réformateurs, dont plusieurs appartiennent d'ailleurs à des conseils d'administration de colonies privées, s'inquiètent soudainement pour la survie des établissements dont ils ont la charge. Ils craignent également l'engorgement des sociétés de patronage et leur invasion par des jeunes trop peu amendables. La colonie leur parait encore l'établissement le mieux adapté pour les jeunes les plus difficiles, ceux pour qui toute mesure charitable semble inadaptée. Il faut voir également dans leur geste une crainte devant la disparition d'un outil répressif, à un moment où, Michelle Perrot l'a montré, la société française prend peur de sa jeunesse. ${ }^{(45)}$ Leur inquiétude, ainsi que celle plus globale de la société française, sauve les colonies correctionnelles, qui apparaissent 
pourtant de mois en moins adaptées à leur mission. Par leur geste, les principaux pourfendeurs des colonies durant les années 1880 finissent par les préserver dans les années 1900. La guerre, peu favorable à l'innovation dans ce secteur à un moment où, à l'arrière, la répression policière se concentre sur les femmes et les jeunes, favorise leur maintien. ${ }^{(46)}$

\section{Conclusion}

Proches de la fermeture à la Belle Époque, quelques colonies privées et publiques franchissent malgré tout la guerre. Leur évolution reprend alors son chemin chaotique. Elles traversent, de la fin de la guerre au début des années 1930, une longue période d'atonie. ${ }^{(47)}$ Les écrits sur le sujet sont alors soit particulièrement peu nombreux, ce qui représente un contraste par rapport aux années précédentes, soit trop aux marges d'un secteur encore dominé par le positivisme juridique et le carcan d'une pensée organisée en fonction du Code pénal. ${ }^{(48)}$ Dans les établissements, les conditions de vie se détériorent encore. La politique d'économie budgétaire des différents gouvernements est menée au détriment des jeunes détenus. Le prix de journée versé pour chaque jeune stagne, alors que l'inflation est forte. Les institutions doivent réaliser des économies sur tout. Les carences du régime alimentaire des jeunes sont particulièrement marquées. Tout manque : vêtements, literie, nourriture, etc. À Mettray, les toitures ne retiennent plus la pluie, les maisons de famille menacent de s'effondrer. Le personnel, souvent issu de l'armée et parfois traumatisé, moralement et physiquement, par la brutalité guerrière, connaît de nombreux problèmes d'alcoolisme et de violence. Les années 1920 sont bien les années noires des colonies agricoles.

Les années 1930 ne sont guère meilleures, même si quelques réformes sont tentées ici ou là. Cependant un nouvel élément intervient : l'opinion. Les colonies privées et publiques deviennent la cible de plusieurs campagnes de presse. La mieux connue de celles-ci, parce que la plus décisive, est celle d'Alexis Danan. Dans des articles d'une extrême virulence, publiés dans des journaux à forts tirages, il dénonce les bagnes d'enfants, des lieux de violence et de malheur. Les arguments de Danan sont repris dans des brochures. Le grand scientifique de sensibilité communiste $\mathrm{H}$. Wallon
Perrot, Les ombres de l'histoire, crime et châtiment au XIXème siècle, Paris, Flammarion, 2001, p. 351-364. Dominique Kalifa, L'encre et le sang. Le récit de crime à la Belle Époque, Paris, Fayard, 1995, 351 p. Dominique Kalifa, "Les Apaches à Paris ", Le magazine littéraire, $n^{\circ} 288$, mai 1991, p. 68.

(46) Par exemple, sur la question du contrôle des mœurs pendant la guerre, Jean-Yves Le Naour, Misères et tourments de la chair durant la Grande Guerre, les moeurs sexuelles de Français 19141918, Paris, Aubier, 2002.

(47) Sur cette période, nous renvoyons à Éric Pierre, " Mettray dans les années 1920, une période noire ", in Raoul Léger, La colonie agricole et pénitentiaire de Mettray. Souvenirs d'un colon 19221927, Paris, L'Harmattan, 1997, p. 127-151. 
(48) Nous pensons aux textes de certains psychiatres.

(49) Henri Wallon, Une plaie de la société : les bagnes d'enfants, Bourges, Secours ouvrier international, 1934.
(50) Henri Gaillac, $o p$. cit., p. 261-357. Gaëlle Guimbretière, La réforme de l'éducation surveillée à Saint-Maurice (19361958), université d'Angers, mémoire de maîtrise, 1999, 171 p.

(51) Il en est tout autrement pour le secteur privé profondément remanié, Michel Chauvière, Enfance inadaptée : l'héritage de Vichy, Paris, Éditions ouvrières, 1980.

(52) Jacques Bourquin,

«Sur la trace des premiers éducateurs de l'Éducation surveillée : 1936-1947 ", Cabiers du CRIV, $\mathrm{n}^{\circ} 2$, octobre 1986, p. 11-60. écrit, lui aussi, une brochure sur les colonies. ${ }^{(49)}$ L'historien est bien évidemment incapable de mesurer les effets d'une telle campagne en terme d'opinion publique. Mais une chose est sûre, elle contraint le gouvernement à réagir. Des établissements sont fermés, d'autres doivent être réformés. Ces premières réformes sont bien connues. ${ }^{(50)}$ Une première fait appel au scoutisme pour former un personnel éducatif. Une autre tente d'instaurer une véritable formation professionnelle pour les jeunes. Elles suscitent toutes deux une grande hostilité du personnel en place. La première échoue rapidement ; la seconde ne réussit à s'imposer que par un changement du personnel de direction, mais elle est loin de toucher à tous les aspects de l'éducation correctionnelle.

La seconde guerre mondiale donne un coup d'arrêt à ce mouvement réformateur qui, dans les établissements, ne reprend qu'au lendemain de la Libération. ${ }^{(51)}$ Le secteur de l'enfance délinquante change alors en profondeur avec des modifications législatives (l'ordonnance de 1945), la naissance d'une administration porteuse de réformes (l'Éducation surveillée qui se sépare de l'Administration pénitentiaire), le recrutement d'un personnel, en principe, formé à sa mission auprès des jeunes. ${ }^{(52)}$ Cependant ce nouveau secteur doit encore gérer le poids du passé en assumant pour héritage les établissements anciens. Le poids des murs pénitentiaires pèse encore lourdement sur les nouvelles politiques de gestion de la délinquance juvénile. Il faut alors de longues années pour tenter encore de réformer ces établissements nés un siècle auparavant, puis finalement décider de les fermer. 